\title{
Effect of Flash Glucose Monitoring in T1DM Patients with Multiple Daily Insulin Injections:Improved HbA1C and Reduced Glycemic Variability
}

\section{Wenhui ZHANG}

Liaocheng People's Hospital

\section{Yu LIU}

Liaocheng People's Hospital

\section{Baosheng SUN}

Liaocheng Veterans Hospital

\section{Yanjun SHEN}

Liaocheng People's Hospital

Ming LI

Liaocheng People's Hospital

\section{Lanbo PENG}

Liaocheng People's Hospital

\section{Honggang DUAN}

Liaocheng Peoples Hospital

\section{Xudong SU}

Liaocheng Peoples Hospital

Shaoxia LU

Liaocheng Veterans Hospital

\section{Xiaoqin TIAN}

Liaocheng Central Hospital

Yaqiang Tian ( $\sim$ tyq13346255990@163.com )

Liaocheng People's Hospital

Original investigation

Keywords: Flash glucose monitoring, Type 1 diabetes, HbA1c, Glycemic variability, Hypoglycemia

Posted Date: June 30th, 2021

DOI: https://doi.org/10.21203/rs.3.rs-657144/v1 
License: (c) (i) This work is licensed under a Creative Commons Attribution 4.0 International License. Read Full License 


\section{Abstract}

Background: Flash glucose monitoring(FGM) was introduced to China in 2016 and it might improve $\mathrm{HbA1C}$ and reduce glycemic variability for T1DM therapy.

Methods: A total of 146 patients were recruited from October 2018 to September 2019 in Liaocheng. Patients were randomly divided into FGM group or self-monitoring blood glucose(SMBG) group. Both groups wore the FGM for multiple 2 week periods, beginning with the 1st, 24th, and 48th week for gathering data, while blood samples were collected for $\mathrm{HbA} 1 \mathrm{c}$ measurement. The dietary guidance and insulin dose adjustment were made in FGM group patients according to the dynamic blood glucose monitoring map as well as SMBG group patients according to SMBG measurements 3-4 times daily. All of the participants would insist on SMBG measurements in the days without wearing the FGM device. At the final visit, $\mathrm{HbA} 1 \mathrm{C}$,time in range(TIR),the duration of hypoglycemia and numbers of diabetic ketoacidosis(DKA) were taken as the main observational indexes.

Results: There was no significant difference in baseline characteristics of the two groups. At 24 week,HbA1c level of FGM gruop was $(8.16 \pm 1.03) \%$,much lower than that of SMBG group $(8.68 \pm 1.01) \%$ $(p<0.05)$.Interquartile range $(I Q R)$, mean blood glucose $(M B G)$,duration of hypoglycemia of FGM group also showed significant decline, compared with SMBG group $(p<0.05)$, while TIR displayed apparently increase in FGM group [(49.39 \pm 17.54$) \%$ vs $(42.44 \pm 15.49) \%](p<0.05)$. At 48 week,the differences were more pronounced $(p<0.01)$. There was no observed change in the number of episodes of DKA when study was finished [(0.25 \pm 0.50$)$ vs $(0.28 \pm 0.51), P \llbracket 0.75]$.

Conclusion: Intermittent use of FGM in T1DM patients can improve HbA1c and glycemic control without increasing hypoglycemic exposure in insulin-treated type 1 diabetes individuals in the undeveloped country.

\section{Introduction}

Type 1 diabetes mellitus(T1DM) is a challenging chronic autoimmune condition resulting in absolute shortage of insulin production. Every year there are an estimated more than 13000 new T1DM patients in China. Most of them are adults treated with multiple daily injection(MDI) ${ }^{[1,2]}$. There are some unique clinical and demographic characteristics in Chinese type 1 diabetes patients, such as poor blood glucose control,extremely low blood glucose monitoring frequency, irregular insulin treatment, frequent acute and chronic complications $\square$ late onset and lean body size ${ }^{[3]}$. It is necessary to achieve favorable glucose level for T1DM patients to prevent diabetes-related complications, including severe hypoglycemia,lifethreatening diabetic ketoacidosis (DKA), macrovascular complications (such as peripheral arterial disease $\square$ coronary heart disease and cerebrovascular disease) and microvascular complications (such as retinopathy $\llbracket$ nephropathy and neuropathy $)^{[4]}$. 
As the basis of modern T1DM treatment,blood glucose monitoring plays an important role for several years. In 1978[self-monitoring blood glucose (SMBG) was first applied to clinical therapy ${ }^{[5]}$. The close relationship between SMBG frequency and improved diabetes control have been confirmed by several studies $^{[6]}$. However, There are many problems with SMBG, including stress cost, pain and technical skills, which led to the development of retrospective continuous glucose monitoring (CGM) and real-time continuous glucose monitoring (rt-CGM) ${ }^{[7]}$. CGM can provide information that SMBG cannot obtain, such as real-time display of glucose level and glucose change rate, and the ability to characterize blood glucose variability ${ }^{[8]}$. Randomized controlled trials have indicated that CGM combined with subcutaneous insulin infusion (CSII) or multiple daily injection (MDI) could increase hemoglobin A1c(HbA1C) level and reduce hypoglycemia ${ }^{[9-12]}$. In the past decades, the management of T1DM has made remarkable progress, partly due to CGM and rt-CGM technology ${ }^{[6,13]}$. Although the accuracy and usability of rt-CGM have been gradually improved, this technology has not been widely adopted so far because of cost and inconvenience ${ }^{[7,8]}$.

In 2014, a new continuous glucose monitoring system-flash glucose monitoring(FGM) was introduced to European Union,and then it was applied in China in $2016^{[14]}$. There are some major differences between FGM and other real- time CGM Systems.FGM can provide ambulatory glucose profile (AGP)for 14 days without blood calibration, receiving much attention of clinicians and diabetic patients.

Our overall goal is to investigate whether the use of FGM could be an effective method by achieving improvement of $\mathrm{HbA} 1 \mathrm{C}$ and reducing glycemic variability as well for T1DM therapy.

\section{Methods}

\section{Study designs and aims}

We carried on a 50-week single-site randomized controlled trial to evaluate the effect of FGM on blood glucose control and variability in T1DM patients with MDI.

\section{Sample Size}

The estimation of the sample size is based on the estimated incidence of T1DM in China and the results of $\operatorname{star}^{[2,15]}$. Decrease of $\mathrm{HbA} 1 \mathrm{c}$ by $0.3 \%$ is generally considered as a clinically significant decrease in order to reduce diabetic complications. With the power of $90 \%$ at $P=0.05$ (two-sided), 72 patients in each group are needed to detect the $0.3 \%$ difference of $\mathrm{HbA} 1 \mathrm{C}$ between both groups. So the study needed 160 patients who were selected from Liaocheng People's Hospital, Liaocheng Veterans Hospital and Liaocheng Central Hospital assuming a dropout rate of $10 \%$.

\section{Population}

We strive to establish a T1DM management model in undeveloped area. Our hospital serves a catchment area of about 10 million people and our group has devoted to the registration and management of 
baseline data for T1DM patients in Liaocheng area in the past six years. We have conducted the prevalence of T1DM in Liaocheng in previous study,which is consistent with that in Guangdong province $^{[3,16]}$. In this study ,all the participants are derived from our management system , who have accepted regular and systemic diabetes education.

\section{Inclusion criteria}

(1) Age $\geq 4$ years old; (2) $\mathrm{HbA} 1 \mathrm{C} \geq 7 \%$; (3) All patients were given multiple daily injection (MDI); (4)with the diagnosis of T1DM at least 3 months.

\section{Exclusion criteria}

(1)patients with severe complications;(2) pregnancy;(3) Application of FGM and/or CSII in the previous 3 months.

This study has applied for registration of clinical trial and the registration number of Chinese clinical trial is ChiCTR-INR-16009665.

\section{Study procedures}

\section{Data collection}

We gathered age, diabetes courses, insulin dosages and calculated BMI of participants.After the HbA1c analysis was done, all of them wore a FGM for 2 weeks. The data including glucose fluctuations, time in range, mean glucose, and hypoglycemia time was downloaded and calculated as the baseline in the 1st week without any intervention. Then patients were randomly divided into FGM group or SMBG group at a ratio of $1: 1$.

\section{Procedures}

Both groups wore the FGM for multiple 2 week periods, beginning with the 1st, 24th, and 48th week for gathering data, while blood samples were collected for HbA1c measurement. We gathered baseline data in the 1st week. In the 2nd week, FGM group received directions on best use of FGM, including meanings of trend arrows and glucose profiles for treatment adjustment, and how to deal with hyperglycemia and hypoglycemia. At the same time, SMBG group insisted measuring 3-4 times blood glucose daily, including assessment of FPG and post-meal measurements. The dietary guidance and insulin dose adjustment were made in FGM group patients according to the dynamic blood glucose monitoring map as well as SMBG group patients according to SMBG measurements 3-4 times daily. From 3 to 23 week,SMBG measurements (3-4 times a day)were persisted and routine care from our management system were received in both groups. Both groups wore the FGM again at 24 to 25 weeks,receiving the same intervention as before for gathering data,while blood samples were collected for HbA1c measurement.Then from 26 to 47 weeks ,all of the participants would insist on SMBG measurements in the days without wearing the FGM device.

\section{Outcomes}


The follow-up phase began at 48 week. All of the participants were required to wear the FGM for 14 days again. The data was downloaded and blood samples were collected for $\mathrm{HbA} 1 \mathrm{c}$ measurement at the final visit. HbA1C,TIR,the duration of hypoglycemia and numbers of diabetic ketoacidosis were taken as the main observational indexes.

\section{Statistical analysis}

SPSS statistical software version 17.0 was used to perform statistical analyzation.Normally distributed continuous data was performed by Mean \pm SD and skewed continuous data was summarized by medians. For categorical data,we used numbers and proportions to analyze. All the data was tested at $5 \%$ significance level. T-test was applied to analyze normally distributed data and Wilcoxon signed rank test was used to analyze skewed continuous data. Besides,chi-squared test was assessed to difference in proportions.

\section{Results}

\section{Clinical characteristics}

A total of 160 patients were recruited from October 2018 to September 2019 in Liaocheng, Shandong province. Because of refusal to participate in the study, 146 subjects( 64 males, 82 females ) were left to be analyzed at baseline.As presented in Table 1, there was no significant difference in baseline characteristics of both groups including age, courses of disease, body mass index and insulin dosages after randomization ( $p>0.05)$. The baseline blood glucose characteristics $(H b A 1 c, T I R, I Q R, M B G$ and duration of hypoglycemia )in the fisrt week without intervention also showed no statistics difference.

Table 1

Baseline characteristics of participants $[\overline{\mathrm{x}} \pm \mathrm{s}, \mathrm{M}(\mathrm{QL} \square \mathrm{QU}) \square \mathrm{n} / \mathrm{n}]$

\begin{tabular}{|llllll|}
\hline Group & $\mathrm{n}(\mathrm{M} / \mathrm{F})$ & Age(Years) & $\begin{array}{l}\text { DM duration } \\
(\text { Years })\end{array}$ & $\begin{array}{l}\text { BMI } \\
\mathbf{( k g / m 2 )}\end{array}$ & $\begin{array}{l}\text { Insulin dosage } \\
(\mathbf{u} /(\mathbf{k g} . \mathbf{d}))\end{array}$ \\
\hline FGM & $71(34 / 37)$ & $36.68 \pm 19.71$ & $4.0(2.0,7.0)$ & $19.70 \pm 2.01$ & $0.68 \pm 0.08$ \\
\hline SMBG & $75(30 / 45)$ & $35.19 \pm 18.91$ & $5.0(2.0,7.0)$ & $19.13 \pm 1.66$ & $0.69 \pm 0.07$ \\
\hline P & 0.34 & 0.64 & 0.67 & 0.06 & 0.43 \\
\hline
\end{tabular}

FGM:Flash glucose monitoring; SMBG:Self-monitoring blood glucose;BMI:body mass index 
Table 2

Baseline blood glucose characteristics of participants $(\overline{\mathrm{x}} \pm \mathrm{s})$

\begin{tabular}{|llllll|}
\hline Group & HbA1C(\%) & TIR(\%) & IQR(mmol/L) & MBG(mmol/L) & $\begin{array}{l}\text { Hypoglycemic } \\
\text { duration(min) }\end{array}$ \\
\hline FGM & $\begin{array}{l}9.05 \pm \\
1.43\end{array}$ & $\begin{array}{l}36.49 \pm \\
17.57\end{array}$ & $8.39 \pm 2.69$ & $11.79 \pm 2.20$ & $201.96 \pm 44.28$ \\
\hline SMBG & $\begin{array}{l}9.07 \pm \\
1.18\end{array}$ & $\begin{array}{l}37.87 \pm \\
15.87\end{array}$ & $8.10 \pm 1.94$ & $11.81 \pm 1.93$ & $198.13 \pm 35.90$ \\
\hline P & 0.95 & 0.62 & 0.46 & 0.95 & 0.57 \\
\hline
\end{tabular}

TIR囚time in range; IQR囚interquartile range; MBG®mean blood glucose

Table 3

Comparison of glycemic parameters at 14-week $(\overline{\mathrm{x}} \pm \mathrm{s})$

\begin{tabular}{|c|c|c|c|c|c|c|}
\hline Group & $\mathrm{n}$ & $\mathrm{HbA1C}(\%)$ & $\operatorname{TIR}(\%)$ & $\begin{array}{l}\text { IQR } \\
\text { (mmol/L) }\end{array}$ & $\mathrm{MBG}(\mathrm{mmol} / \mathrm{L})$ & $\begin{array}{l}\text { Hypoglycemic } \\
\text { duration(min) }\end{array}$ \\
\hline FGM & 71 & & & & & \\
\hline $1 \mathrm{~W}$ & & $9.05 \pm 1.43$ & $\begin{array}{l}36.49 \pm \\
17.57\end{array}$ & $8.39 \pm 2.69$ & $11.79 \pm 2.20$ & $201.96 \pm 44.28$ \\
\hline $24 W$ & & $8.16 \pm 1.03$ & $\begin{array}{l}49.39 \pm \\
17.54\end{array}$ & $6.85 \pm 2.35$ & $10.48 \pm 1.63$ & $175.86 \pm 43.19$ \\
\hline $48 W$ & & $7.39 \pm 0.71$ & $\begin{array}{l}62.35 \pm \\
12.29\end{array}$ & $5.70 \pm 2.03$ & $9.21 \pm 1.18$ & $158.78 \pm 35.31$ \\
\hline SMBG & 75 & & & & & \\
\hline $1 \mathrm{~W}$ & & $9.07 \pm 1.18$ & $\begin{array}{l}37.87 \pm \\
15.87\end{array}$ & $8.10 \pm 1.94$ & $11.81 \pm 1.93$ & $198.13 \pm 35.90$ \\
\hline $24 w$ & & $\begin{array}{l}8.68 \pm \\
1.01^{\star}\end{array}$ & $\begin{array}{l}42.44 \pm \\
15.49^{\star}\end{array}$ & $\begin{array}{l}7.57 \pm \\
1.81 *\end{array}$ & $11.22 \pm 1.60 *$ & $191.95 \pm 37.11$ * \\
\hline $48 w$ & & $\begin{array}{l}8.36 \pm \\
1.02^{\star \star \star}\end{array}$ & $\begin{array}{l}46.52 \pm \\
16.65^{\star \star}\end{array}$ & $\begin{array}{l}7.10 \pm \\
1.91^{\star \star}\end{array}$ & $10.78 \pm 1.62^{\star \star}$ & $192.80 \pm 31.08$ *夫 \\
\hline
\end{tabular}

TIR囚time in range; IQR囚interquartile range; MBG®mean blood glucose

*:Compared with FGM at 24W,p<0.05;**: Compared with FGM at 48W,p<0.01.

\section{Comparison of glycemic parameters at 24-week and 48- week follow-up}

At 24 week,HbA1c level of FGM gruop was $(8.16 \pm 1.03) \%$,much lower than that of SMBG group $(8.68 \pm 1.01) \%(p<0.05)$. IQR,MBG, duration of hypoglycemia of FGM group also showed significant 
decline, compared with SMBG group $(\mathrm{p}<0.05)$, while TIR displayed apparently increase in FGM group $[(49.39 \pm 17.54) \% v s(42.44 \pm 15.49) \%](p<0.05)$. At 48 week,the differences were more pronounced $(p<0.01)$.

For FGM group, the HbA1c concentration decreased from 9.05区 at baseline to 8.16》 at 24 week $(p<0.05)$ and $7.39 \square$ after 48 weeks $(p<0.05)$, resulting in an overall difference in HbA1c over the study period.At the same time,IQR,MBG,duration of hypoglycemia also displayed marked decrease at 24 week and 48 week $(p<0.05)$.TIR increased apparently from $36.49 \rrbracket$ to $49.39 \rrbracket$ at 24 week to $62.35 \rrbracket$ at the end of study $(p<0.05)$.

In addition, there was no observed change in numbers of DKA when study was finished $[(0.25 \pm 0.50)$ vs (0. 28 \pm 0.51$), P \llbracket 0.75]$.

\section{Discussion}

T1DM is an endocrine metabolic disease in which pancreas islet $\beta$ cells are destroyed by the autoimmune system and insulin therapy are required for life. The management of chronic hyperglycemia, hypoglycemia and blood glucose variability in T1DM is the difficulty in clinical treatment at present ${ }^{[17]}$.Monitoring of glucose levels is essential for achieving target glycemic control and avoiding hypoglycemia, especially in patients with T1DM.

As a new technology,flash glucose monitoring (FGM) has recently been accepted by clinicians rapidly instead of CGM/SMBG. In FGM, glucose data could be stored for up to 8 hours on a sensor and a handheld reader could be used to obtain them conveniently.Moreover, the FGM sensor is factorycalibrated and can be worn for up to 14 days,popular with patients requiring high qualities of life [18].

In this study we aimed to identify whether using FGM can improve glycemic control among patients with T1DM in Liaocheng district.

The 2017 "International Consensus of Continuous Glucose Monitoring" emphasized that the "three core indicators" of FGM monitoring are TIR, blood glucose variability, and hypoglycemia .In 2019,Marion Fokkert collected daily life data from persons with DM using the FGM system $\square$ finding that after 1-year follow-up $\square \mathrm{HbA} 1 \mathrm{c}$ declined from $64 \mathrm{mmol} / \mathrm{mol}$ to $60 \mathrm{mmol} / \mathrm{mol}$. While, patients reported less and less severe hypoglycemias and a more active role toward treatment ${ }^{[19]}$.Study of Ramzi A Ajjan also displayed similar results. They found that FGM can improve $\mathrm{HbA} 1 \mathrm{c}$ and treatment satisfaction without increasing hypoglycemic exposure in insulin-treated type 2 diabetes individuals managed in primary/secondary care centres ${ }^{[20]}$.The DIAMOND and GOLD studies showed the benefit of CGM in people using conventional MDI treatment as well, which the majority of patients with T1DM use ${ }^{[12,21]}$.

In our study,HbA1C was $9.05 \%$ at baseline,demonstrating T1DM patients' poor glycemic control in Liaocheng,while it decreased largely after intermittent use of FGM at 48 week(from $9.05 \rrbracket$ to $7.39 \square)(p<$ $0.05)$,similar with above studies. At the same time, hypoglycemic duration does not increase. Instead,it 
decreased sharply at the end of study, which demonstrated using FGM was able to improve HbA1C without increase of hypoglycemic time.

Blood glucose variability, also known as blood glucose fluctuations, is an unstable state in which blood glucose levels change between peaks and troughs. IQR is considered the most appropriate value for expressing blood glucose variability ${ }^{[22]}$. The results of a large randomized clinical trial known as IMPACT demonstrated that there was apparent improvements in time in range and glucose variability in patients who were randomized to flash $\mathrm{CGM}^{[23]}$. In our research,we also described obvious improvement in TIR at 48 week and reduced IQR,which meant patients of intervention group got good glycemic control and reduced blood glucose variability.

Study of Dunn et al. discovered in real-world conditions, flash glucose monitoring with higher rates of scanning linked to improved glycemic markers, including increased time in range and reduced estimated $\mathrm{HbA1c}$ from $8.0-6.7 \%{ }^{[24]}$. Research of Deng mingqun also showed that the FGM-derived TIR could be helpful in the glucose management of Chinese adult T1DM, while glucose variability should be taken into consideration in interpreting the relationship between $\mathrm{TIR}$ and $\mathrm{HbA} 1 \mathrm{c}^{[25]}$. Our study got similar results that after intermittent FGM intervention, the TIR value increased from $36.49-62.35 \%$ at 48 week, and the HbA1C fell up to $7.39 \%$.

With regard to safety, no sensor-related adverse events occurred and the few events such as insertion and shedding problems were similar to the events observed in other researches $[22,26]$.

Lots of researches displayed that estimating glycemic control from $\mathrm{HbA} 1 \mathrm{c}$ alone is in essence applying a population average to an individual, which can be misleading ${ }^{[27]}$. Flash glucose monitoring system can provide real-time interstitial glucose levels and trends of glucose levels. Moreover, it has the advantage of being factory calibrated so the user does not have to do any $\mathrm{CBG}^{[28]}$. Beyond that, users could acquire a patient's CGM glucose profile, which has considerable value for optimizing diabetes management.

Previous studies all focused on the influence of continuous application of FGM for T1DM patients.In China,it's the first time to discuss the effects of 1-year intermittent use of FGM in T1DM patients, which may be helpful to follow-up studies and application of FGM for diabetes prevention and blood glucose management.

This study has a number of limitations,too. First of all, participants have to realize the intervention, so the research could not be blinded, which may affect the results. Secondly, the intermittent intervention could underestimate the potential benefit of FGM.Thirdly, as data were patient-reported, recall bias may be present.Finally, the current population was a selection of the patients in Liaocheng district, which may implicate selection bias.

\section{Conclusion}


In summary, intermittent use of FGM in T1DM patients can improve HbA1c and glycemic control without increasing hypoglycemic exposure in insulin-treated type 1 diabetes individuals in the undeveloped country.

\section{Abbreviations}

FGM: Flash glucose monitoring, T1DM: Type 1 diabetes, SMBG: self-monitoring blood glucose, TIR: time in range, DKA: diabetic ketoacidosis, IQR: Interquartile range, MBG: mean blood glucose, MDI: multiple daily injection, CGM: continuous glucose monitoring, rt-CGM: real-time continuous glucose monitoring, CSII: subcutaneous insulin infusion, HbA1C: hemoglobin A1c, AGP: ambulatory glucose profile.

\section{Declarations}

\section{Ethics approval and consent to participate}

The clinical research program was approved by Ethics Committee of Liaocheng People's Hospital. Written and verbal informed consent were demanded to provide in all participants.

\section{Consent for publication}

All authors agree to submit the manuscript for publication in Cardiovascular Diabetology.

\section{Availability of data and materials}

We have uploaded raw data as supplementary materials.

\section{Competing interests}

The authors have no conflicts of interest relevant to this article to disclose.

\section{Funding}

This study was supported by Shandong Medical and Health Science and Technology Project (2015WS0392).

\section{Authors' contributions}

All authors have contributed significantly in this manuscript. Wenhui Zhang drafted the initial manuscript. Yaqiang Tian conceptualized and designed the study. Yu Liu and Baosheng Sun carried out the initial analysis. Yanjun Shen and Xudong Su reviewed and revised the manuscript. Ming Li,Lanbo Peng and Honggang Duan helped to conduct statistical analysis and data collection. Shaoxia Lu and Xiaoqin Tian coordinated and supervised data collection at two other sites and critically reviewed the manuscript.

\section{Acknowledgements}




\section{References}

1. Miller KM, Beck RW, Bergenstal RM, et al. Evidence of a strong association between frequency of selfmonitoring of blood glucose and hemoglobin A1c levels in T1D exchange clinic registry participants. Diabetes care. 2013,36(7):2009-14.

2. Weng J, Zhou Z, Guo L, et al. Incidence of type 1 diabetes in China, 2010-13: population based study. BMJ (Clinical research ed). 2018,360:j5295.

3. Li J, Chen D, Wu J, et al. Guangdong T1DM translational medicine study (1):Clinical characteristics of 3159 type 1 diabetic patients. Asian Association for the Study of Diabetes,AASD2011. p. 25-6.

4. Diagnosis and classification of diabetes mellitus. Diabetes care. 2010,33 Suppl 1:S62-9.

5. Walford S, Gale EA, Allison SP,et al. Self-monitoring of blood-glucose:Improvement of diabetic control. Lancet (London, England). 1978,1(8067):732-5.

6. Lawson ML, Bradley B, McAssey K, et al. The JDRF CCTN CGM TIME Trial: Timing of Initiation of continuous glucose Monitoring in Established pediatric type 1 diabetes: study protocol, recruitment and baseline characteristics. BMC pediatrics. 2014,14:183.

7. Rodbard D. Continuous Glucose Monitoring: A Review of Successes, Challenges, and Opportunities. Diabetes technology \& therapeutics. 2016,18 Suppl 2:S3-s13.

8. Blevins TC, Bode BW, Garg SK, et al. Statement by the American Association of Clinical Endocrinologists Consensus Panel on continuous glucose monitoring. Endocrine practice. 2010,16(5):730-45.

9. Hirsch IB, Armstrong D, Bergenstal RM, et al. Clinical application of emerging sensor technologies in diabetes management: consensus guidelines for continuous glucose monitoring (CGM). Diabetes technology \& therapeutics. 2008,10(4):232-44, quiz 45-6.

10. Tamborlane WV, Beck RW, Bode BW, et al. Continuous glucose monitoring and intensive treatment of type 1 diabetes. The New England journal of medicine. 2008,359(14):1464-76.

11. Vigersky RA. The benefits, limitations, and cost-effectiveness of advanced technologies in the management of patients with diabetes mellitus. Journal of diabetes science and technology. 2015,9(2):320-30.

12. Lind M, Polonsky W, Hirsch IB, et al. Continuous Glucose Monitoring vs Conventional Therapy for Glycemic Control in Adults With Type 1 Diabetes Treated With Multiple Daily Insulin Injections: The GOLD Randomized Clinical Trial. Jama. 2017,317(4):379-87.

13. Battelino T, Phillip M, Bratina $\mathrm{N}$,et al. Effect of continuous glucose monitoring on hypoglycemia in type 1 diabetes. Diabetes care. 2011,34(4):795-800.

14. Danne T\Nimri R囚Battelino T囚et al. International consensus on use of continuous glucose monitoring. Diabetes Care $₫ 2017 \rrbracket 40 \rrbracket 1631 \rrbracket 1640$. 
15. Slover RH, Welsh JB, Criego A, et al. Effectiveness of sensor-augmented pump therapy in children and adolescents with type 1 diabetes in the STAR 3 study. Pediatric diabetes. 2012,13(1):6-11.

16. Shen Y, Tian y, Li M, et al. Applicaton of real-time continuous glucose monitoring in outpatient management system for type 1 diabetes mellitus. Chinese journal of endocrinology and metabolism. 2017,33(5):367-71.

17. Liu W囚Cai X囚Zhang R囚et al. Clinical factors associated with glycemic variability analyzed by flash glucose monitoring in type 1 diabetes mellitus. Chin J Diabetes $₫$ April 2020『Vol. 28『No. 4

18. EVANS M.Current methods of assessing blood glucose control in diabetes[J].Br J Diabetes,2016,16(Suppl 1):S7-S9.

19. Fokkert M, Dijk P, Edens M, et al. Improved well-being and decreased disease burden after 1-year use of flash glucose monitoring (FLARE-NL4).BMJ Open Diab Res Care 2019,7:e000809.

20. Ajjan RA, Jackson $\mathrm{N}$ and Thomson SA. Reduction in $\mathrm{HbA1}$ c using professional flash glucose monitoring in insulin-treated type 2 diabetes patients managed in primary and secondary care settings: A pilot, multicentre, randomised controlled trial.Diabetes \& Vascular Disease Research2019, Vol. 16(4) 385-395.

21. Beck RW, Riddlesworth T, Ruedy K, et al.Effect of continuous glucose monitoring on glycemic control in adults with type 1 diabetes using insulin injections: the DIAMOND randomized clinical trial. JAMA 2017,317:371-378.

22. Shen $Y \otimes W a n g B$, Li L\et al.Flash glucose monitoring system can reduce glucose volatility in patients with type 1 diabetes mellitus. Chin J Diabetes, November 2020, Vol. 28, No. 11.

23. Bolinder J, Antuna R, Geelhoed $P$, et al. Novel glucose-sensing technology and hypoglycaemia in type 1 diabetes: a multicentre, non-masked, randomised controlled trial . Lancet 2016, 388: 2254-2263.

24. Dunn TC, Xu Y, Hayter G, et al. Real-world flash glucose monitoring patterns and associations between self-monitoring frequency and glycaemic measures: a European analysis of over 60 million glucose tests. Diabetes Res Clin Pract 2018, 137:37-46.

25. Deng M, Zhou L, Zhai X, et al. Relationship between Time in Range Derived from Flash Glucose Monitoring System and HbA1c . Medical Journal of Peking Union Medical College Hospital .ISSN $1674-9081, \mathrm{CN} 11-5882 / \mathrm{R}$.

26. Beck RW, Riddlesworth TD, Ruedy K, et al. Continuous glucose monitoring versus usual care in patients with type 2 diabetes receiving multiple daily insulin injections. A randomized trial. Ann Intern Med 2017, 167: 365-374.

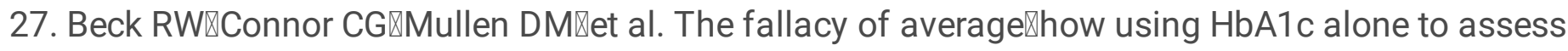
glycemic control can be misleading. Diabetes Care®2017, 40区994\999.

28. Slattery D囚Choudhary P. Clinical use of continuous glucose monitoring in adults with type 1 diabetes. Diabetes Technology\& Therapeutics, 2017, 19® S55『S61.

\section{Supplementary Files}


This is a list of supplementary files associated with this preprint. Click to download.

- 24Wand48W.xlsx

- baselinecharacteristics.xlsx 